\title{
Gradhiva
}

GRADHIV

Revue d'anthropologie et d'histoire des arts

$8 \mid 2008$

Mémoire de l'esclavage au Bénin

« Nos grands-pères achetaient des esclaves... »

Le culte de Mami Tchamba au Togo et au Bénin

"Our grandfathers used to buy slaves..." The cult of Mami Tchamba in Togo and Benin

Alessandra Brivio

(2) OpenEdition

Journals

Édition électronique

URL : https://journals.openedition.org/gradhiva/1188

DOI : 10.4000/gradhiva. 1188

ISSN : 1760-849X

Éditeur

Musée du quai Branly Jacques Chirac

Édition imprimée

Date de publication : 15 novembre 2008

Pagination : 64-79

ISBN : 978-2-915133-94-3

ISSN : 0764-8928

Référence électronique

Alessandra Brivio, « « Nos grands-pères achetaient des esclaves... » », Gradhiva [En ligne], 8| 2008, mis en ligne le 15 novembre 2011, consulté le 21 septembre 2021. URL : http://journals.openedition.org/ gradhiva/1188; DOI : https://doi.org/10.4000/gradhiva.1188 


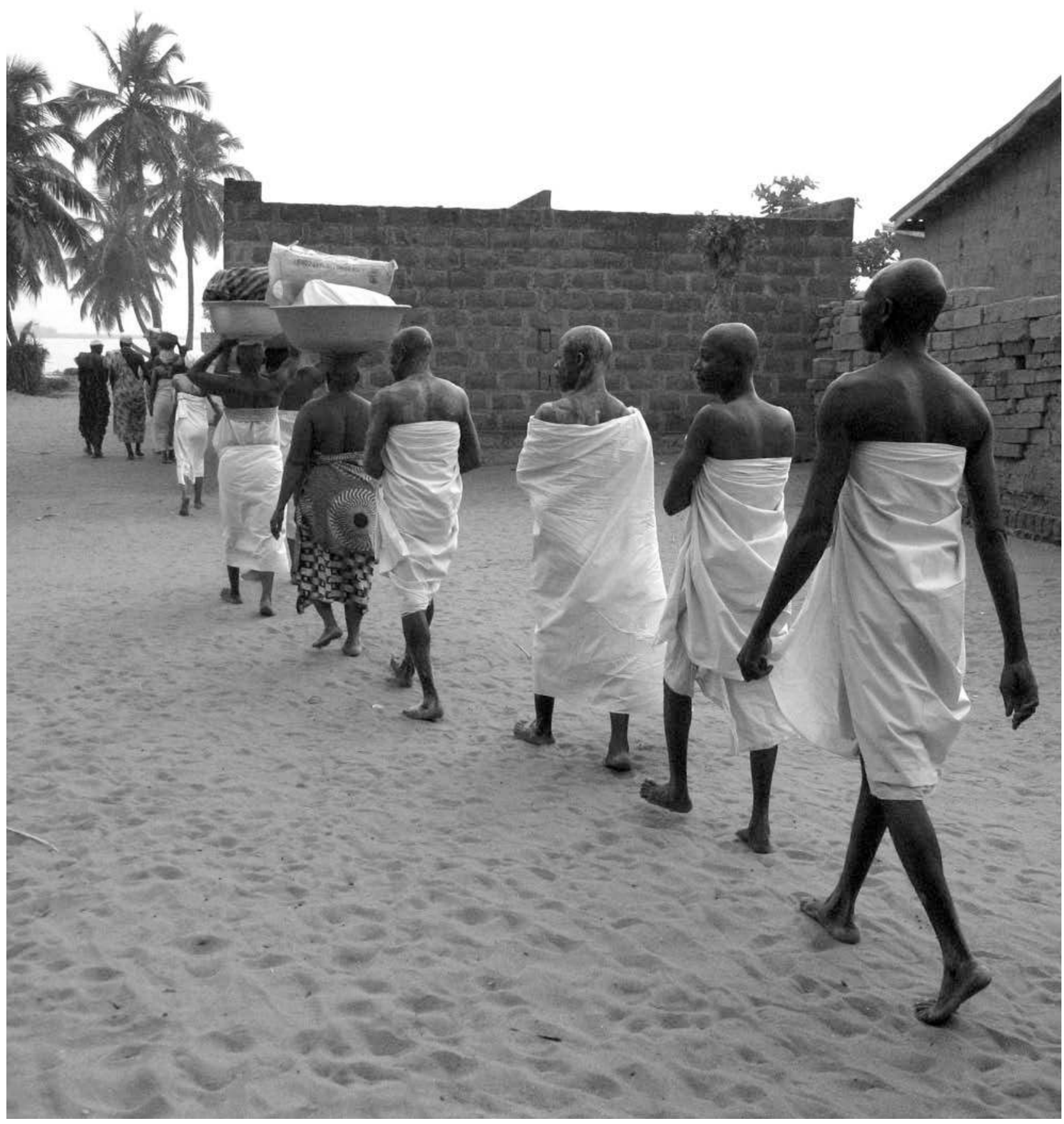

Fig. 1 Les initiés de Tchamba partent au dzogbe à Grand-Popo, Bénin, 2006. Photo A. Brivio. 


\section{« Nos grands-pères achetaient des esclaves... »}

Le culte de Mami Tchamba au Togo et au Bénin*

$\mathrm{T}$ chamba, ou Mami Tchamba, est un des cultes dits vodun répandus dans les régions méridionales du Bénin et du Togo, où ce terme peut désigner à la fois les entités sacrées et les pratiques dont elles sont les destinataires. Au cours des années 1930, Bernard Maupoil, en esquissant un tableau des significations de ces dernières, remarquait : « Nous ignorons le détail de leur origine, presque toujours extérieure au BasDahomey. Telles divinités ont été prises ou achetées à l'ennemi ou au voisin, en Nigeria, en Gold-Coast ou au Togo " (Maupoil 1943 : 52).

À côté de la dimension politique et historique, que Maupoil mit en lumière le premier, il y a une réalité quotidienne, vécue dans une liaison d'intimité et de passion corporelle'. La possession constitue le moment de communion le plus important entre les hommes, les femmes et le monde invisible - les "divinités ${ }^{2}$ ». Les vodun, en fait, loin d'être des réalités transcendantes, expriment l'immanence d'un désir fait de matière, de mots et de liaisons (Augé 1988). Ils s'adaptent, hier comme aujourd'hui, aux espoirs, peurs et interrogations des individus.

Pour comprendre la pratique du vodun et ses implications morales et politiques, on doit souligner son ambiguité ontologique, qui s'inscrit dans un monde où il n'y a pas de place pour une vision dichotomique du réel. Dans un contexte rituel et symbolique où s'imbriquent sans cesse les limites entre le danger et son contrôle, on confère aux vodun en tant qu'agents de mort et de souffrance, mais aussi de protection et de bienfaisance, des qualités ambivalentes qui font l'objet de réaménagements constants de la part des chefs et de leurs adeptes. Cette ambivalence " éthique " peut aider à suivre les trajectoires du culte Tchamba.

Présent parmi les populations de langue ewe et mina des aires côtières du Ghana sud-oriental, du Togo et du Bénin, Tchamba est considéré comme un vodun des esclaves (amèflefle), censé accueillir les esprits de ceux qui sont morts en captivité, loin de leur terre et de leurs divinités. Ces esprits ont choisi les descendants de leurs anciens maîtres pour entretenir l'autel de Tchamba, et leurs corps pour les posséder pendant les cérémonies. En effet, Tchamba est le lieu ou les esprits des esclaves et ceux des maîtres se rencontrent. Sur l'autel, on célè-

\footnotetext{
* Ce texte est le résultat d'une recherche menée dans le cadre du projet inter-universitaire MEBAO, Mission ethnologique au Bénin et en Afrique occidentale, dirigé par Alice Bellagamba et financé par le ministère italien des Affaires étrangères, Direction générale pour la promotion et la coopération culturelle (DGPCC) et cofinancé par le Département de sciences humaines de l'université Milano-Bicocca.

1. À cet égard, il me semble intéressant de rappeler l'échange entre Maupoil et un de ses informateurs : « Jusqu'à quel point votre propre désir ne le crée-t-il pas [le vodun] ? Un vieillard nous répondit : "L'objet du désir, tel est le vodu de chacun." » (Maupoil 1943 : 61).

2. En m'appuyant sur ses usages locaux, j'utiliserai comme équivalents les termes vodun et « divinité ». Pour une analyse des diverses interprétations anthropologiques de la notion, voir Le Hérissé 1911; Herskovits 1933; Maupoil 1943; Augé 1988; Lovell 2002.
} 


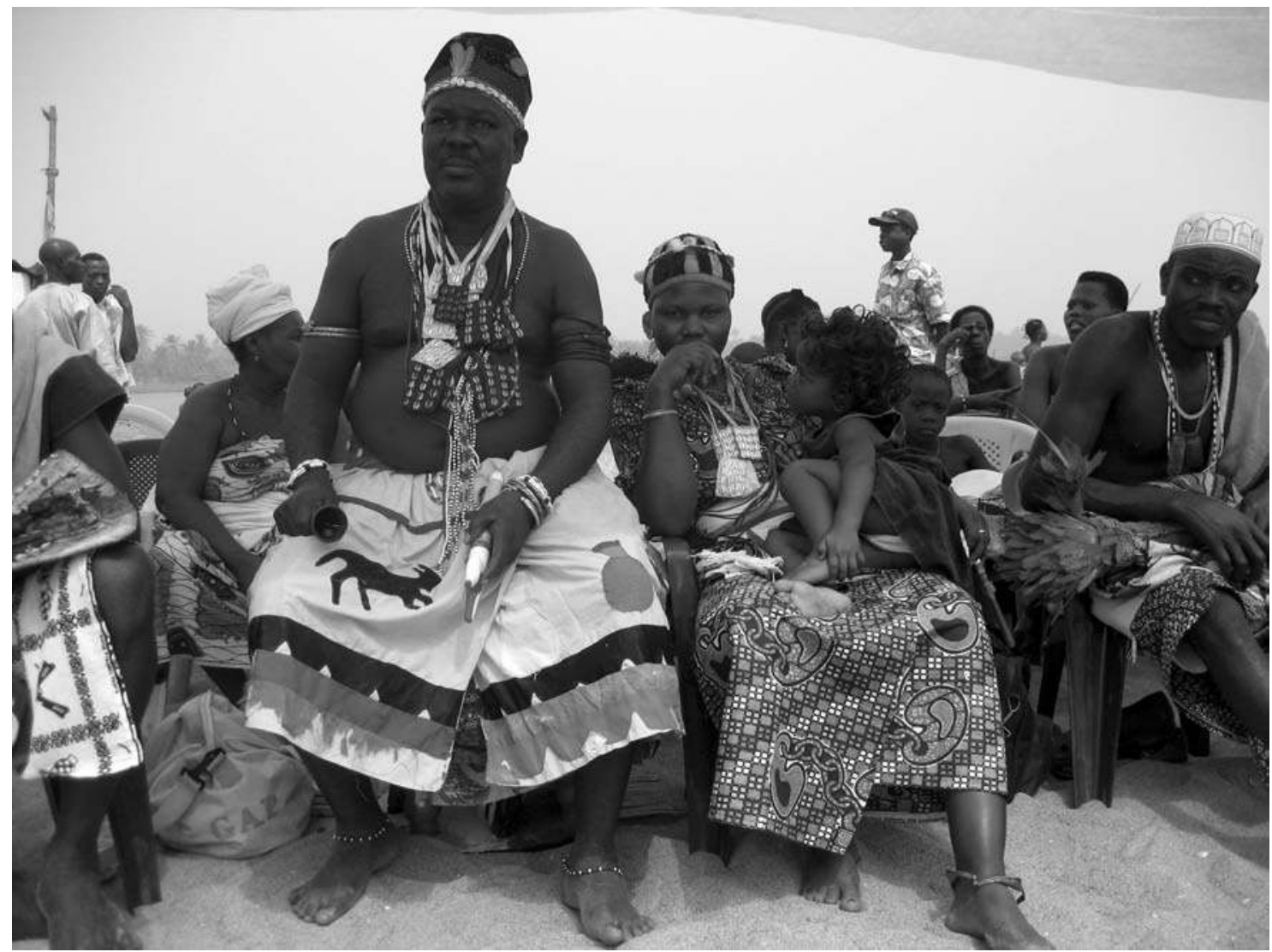

Fig. 2 Dignitaires lors de la fête nationale du vodun du 10 janvier 2006, Grand-Popo, Bénin. Photo A. Brivio.

bre soit les ancêtres impliqués dans le commerce des esclaves, soit leurs victimes. Mais l'ambiguité du vodun Tchamba ne s'arrête pas là, les esclaves étant aussi parfois devenus des ancêtres, par exemple dans le cas des femmes esclaves devenues des épouses. Ici, la limite entre victimes et dominants est floue. Au cours des cérémonies, les corps se mêlent, et le culte excède la sphère familiale et accède à la sphère sociale pour consacrer les esprits de tous les esclaves et de tous ceux qui sont morts loin de leurs terres. Le vodun Tchamba est d'ailleurs proche et souvent mêlé au vodun Ade, consacré aux chasseurs et à tous les hommes et les femmes décédés de "mort violente ", qui n'ont pas reçu de rituels funéraires. Selon les fidèles de Tchamba, il s'agit surtout, dans de tels cas, d'apaiser des esprits " chauds » et agressifs et de détourner leur puissance pour le bien de la famille.

Le culte Tchamba représente une autre façon de rappeler l'esclavage, en dehors des pratiques discursives et des programmes institutionnels inhérents à ses commémorations officielles. Les objets du culte, notamment, portent les traces de cette histoire. Elle peut aussi se transmettre à travers les pratiques de la vie quotidienne et rituelle ainsi que dans les corps qui "vivent " la mémoire (Comaroff 1992; Shaw 2002). Je me propose donc de présenter les résultats d'une enquête sur les imaginaires opaques à l'intérieur desquels les fidèles de Tchamba agissent. Il s'agit de lieux du sacré remémorant un passé de mort et de privation, mais aussi de passion et de désir de richesse.

\section{Des mémoires difficiles}

En Afrique occidentale, l'esclavage représente un passé moralement et socialement difficile à assumer. D'après Ralph Austen, les récits relatifs à cette histoire sont rares à cause des troubles que toute tentative de sa réminiscence provoque. Les mémoires de cette époque n'ont pas disparu, mais elles ont intégré des formes alternatives de narration (Austen 2001). Parmi les auteurs qui ont le plus contribué à la compréhension de cette problématique, Rosalind Shaw, dans son étude sur les communautés de langue temne de la Sierra Leone, a montré la manière dont ces mémoires, avec les trauma- 
tismes historiques et politiques qui leur sont afférents, incorporent des références dérangeantes qu'on peut retrouver, par exemple, dans certaines légendes évoquant des léopards anthropomorphes ou des villes habitées par des sorcières mangeuses d'êtres humains (Shaw 2002). Nicolas Argenti, dans les Grassfields, au Cameroun, a analysé les danses masquées effectuées par les jeunes en tant qu'instruments évoquant la mémoire silencieuse de l'esclavage et du travail forcé; de telles danses ne puisent pas seulement dans le réservoir du passé mais aussi dans la difficile et dramatique liaison hégémonique entre les anciennes et les jeunes générations (Argenti 2006). Sandra Greene, au contraire, a souligné, chez les Anlo-Ewe du Ghana, la nécessité d'analyser les phrases à peine murmurées ou les silences qui ponctuent les narrations communautaires autour de l'esclavage et du savoir religieux (2003). Les traces de l'histoire de l'esclavage persistent aussi dans les pratiques quotidiennes et rituelles (Pietz 1985; Comaroff 1992). Suzanne Preston Blier, dans son étude sur les objets vodun, a montré comment les « fétiches " peuvent être compris comme les réceptacles de la violence du passé : les chaînes, les cordes, les cadenas, serrés autour du « corps » des fétiches, sont des supports mystiques à travers lesquels les officiants du rituel peuvent s'approprier des énergies en évoquant la violence d'antan, exercée par des hommes envers d'autres hommes (Preston Blier 1995).

L'aire géographique où le culte Tchamba s'est répandu, celle de la langue ewe-mina, a été affectée par le commerce des esclaves. L'économie régionale s'organisait autour de la traite atlantique ou de la production d'huile de palme. De nos jours, les pratiques liées au vodun Tchamba évoquent cette histoire d'une façon très différente de celle des discours politiques officiels ou des programmes commémoratifs institutionnels. Durant les cérémonies consacrées à Tchamba, une mémoire physique de l'esclavage s'incarne dans certains des officiants du rite. Ceux qui sont alors considérés comme les esprits des hommes achetés autrefois pénètrent dans le corps des descendants de leurs anciens maîtres, parmi lesquels on retrouve les adeptes du vodun. La relation de ces derniers avec Tchamba est scandée au quotidien

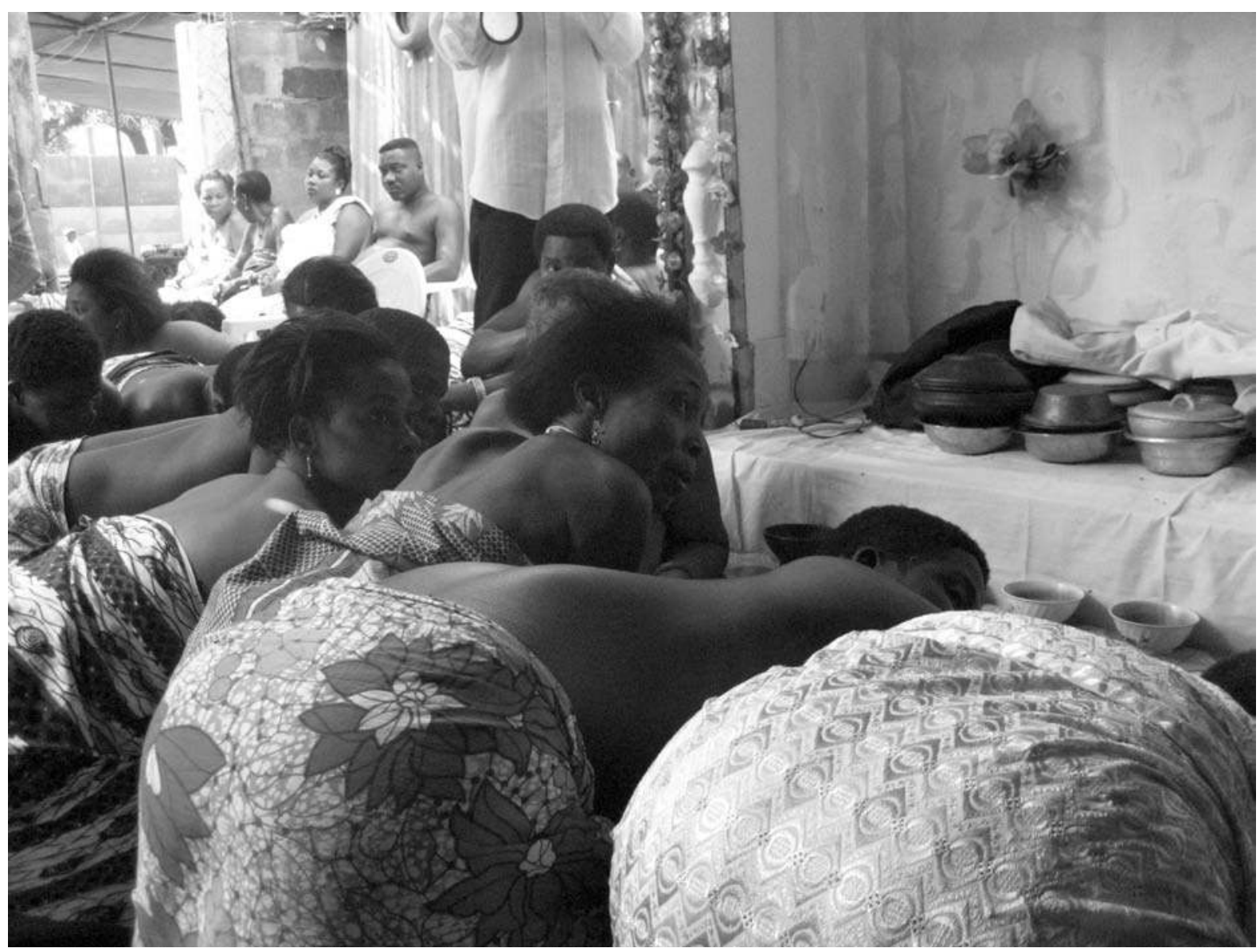

Fig. 3 La prière des adeptes de Tchamba, Lomé, Togo, 2006. Photo A. Brivio. 
par une discipline faite d'objets, de nourritures, de pratiques, dont la transe, mise en scène de la " possession » d'une histoire qui reste non écrite et difficilement dicible.

En ce sens, il ne s'agit pas de résoudre analytiquement une opposition entre raison et passion, entre esprit et corps, mais plutôt d'imaginer un continuum entre les deux et de rappeler l'existence d'une mémoire incorporée dans les pratiques rituelles. Cette mémoire a été oubliée par les procédures de récupération idéologique de l'esclavage qui, à partir du début des années 1990, ont été encouragées par les pays d'Afrique occidentale. Au Bénin, le débat autour de l'esclavage a été à l'origine du premier Festival des arts et de la culture vodun Ouidah 92, du lancement, en 1994, du colloque et du programme de La Route de l'esclave, ainsi que du Sommet du pardon, dans le cadre du programme Réconciliation et développement, en 199933. Selon Nassirou Bako-Arifari, grâce à ces initiatives la mémoire de l'esclavage a « cessé d'être un tabou dans le débat politique national " (2000 : 229) et, en suivant une logique patrimonialisatrice, elle est devenue partie intégrante d'une stratégie politique et touristique. En même temps, au Bénin, on a tenté de conjuguer la fabrication d'une " tradition " vodun en tant que patrimoine immatériel censé relier les diverses mémoires de l'esclavage, qui devaient agir tel un facteur de communion entre deux entités imagées : l'Afrique et la diaspora.

À travers le culte Tchamba, d'autres imaginaires procédant du passé de l'esclavage semblent faire surface. Dans ce contexte, en marge des célébrations mémorielles officielles, le souvenir d'un territoire violenté par l'histoire peut avoir comme contrepoids paradoxal des rituels festifs qui semblent évoquer un « âge d'or » révolu. Toujours en équilibre sur le fil d'un jugement moral ambivalent, les narrations des adeptes du culte semblent aujourd'hui chercher en permanence un compromis entre les discours institutionnels et la mémoire dont leurs corps sont, par moments, les réceptacles.

À la différence des narrations de la traite et de l'esclavage les plus fréquemment analysées par les anthropologues, lesquelles proviennent des dominés d'autrefois (Boddy 1989; Piot 1999; Shaw 2002), le culte Tchamba exprime la " conscience historique " des marchands d'esclaves (Wendl 1999). La compréhension de la perception que les adeptes de ce culte, n'étant pas nécessairement les descendants des esprits des victimes qui les possèdent au cours du rituel, ont de leur généalogie est fondamentale afin de saisir les significations de leur ambivalence morale et de leur gestion psychologique de cette singulière inscription dans l'histoire. Les adeptes de Tchamba que j'ai rencontrés revendiquent leur appartenance à l'élite des marchands, tout en reconnaissant souvent la présence d'une ancêtre esclave dans leur famille. Leur position se situe donc à la limite de cette double identification qui peut être acceptée selon divers niveaux de conscience et/ou de détachement. En ce sens, le culte tente de recoudre une déchirure sociale subie par l'esclave en réintégrant son esprit à l'intérieur d'un cycle de vie et de mort qui le libère de la condamnation à l'oubli héritée de son sort de captif décédé loin de ses divinités protectrices.

\section{Maîtres, esclaves et vodun Tchamba}

La société mina actuelle a commencé à se structurer à partir du XVII siècle, suite à des migrations de populations ga, fante, anlo et adangbe fuyant la région que les Européens appelaient à l'époque " Gold Coast ». Ces groupes de fugitifs s'installèrent dans le territoire environnant d'Aného, déjà occupé par des Peda, et fondèrent Glidji, dont leur royaume prit le nom ${ }^{4}$. Au cours du xviII ${ }^{e}$ siècle, les Guin-Mina furent impliqués dans le commerce des esclaves qui, avec celui de l'ivoire, devint un des facteurs de croissance d'Aného. Dans cette ville, les embarcations provenant de la Gold Coast, étaient prises en charge par des marins d'Elmina qui les emmenaient, à travers la lagune, jusqu'à Ouidah. Produit à ses débuts par la demande d'un marché éminemment africain, le succès de leurs activités économiques permit aux GuinMina d'imposer leur domination sur un certain nombre de villages avoisinants. Toutefois, leur système politique demeurait peu centralisé. Le contrôle militaire de l'ensemble guin-mina, au moment de son expansion la plus importante, investissait le territoire côtier allant du fleuve Mono, qui longe la frontière actuelle entre le Togo et le Bénin, jusqu'à la ville anlo-ewe de Keta au

\footnotetext{
3. À partir des années 1990, ces projets ont eu pour objectif la promotion touristique. Au Ghana, par exemple, l'Emancipation Day, commémorant l'abolition de l'esclavage dans les colonies britanniques, a été transformé sous l'égide du ministère du Tourisme en un événement d'inspiration panafricaniste censé attirer durant les vacances d'été les touristes se reconnaissant dans la diaspora en les impliquant dans diverses manifestations politiques et culturelles.

4. Les territoires guin-mina sont habités par une population très hétérogène formée de Pla, Peda, Adja, Ouatchi, Fon, ainsi que par des AfroBrésiliens, c'est-à-dire à la fois les descendants des anciens esclaves affranchis revenus du Brésil à partir de la première moitié du XIX ${ }^{\mathrm{e}}$ siècle et des métis (Mignon 1985). Les Gan, appelés aujourd'hui Guin, étaient originaires de la région d'Accra, les Fanti, d'Elmina, les Anlo et les Adangbe, de la région du Volta. Le terme « Mina » a été d'abord utilisé pour identifier les Fanti ; par la suite, son usage s'est généralisé.
} 


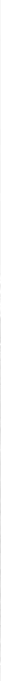

Fig. 4 Le défilé de la fête nationale du vodun du 10 janvier 2006, Grand-Popo, Bénin. Photo A. Brivio.

Ghana (de Medeiros 1984; Law 1991). Dans cet espace politique à l'intérieur duquel Aného, Aflao et Keta étaient les principaux ports impliqués dans la traite négrière, à une échelle réduite par rapport aux formations politiques fon et ashanti voisines, l'esclavage se structura comme une institution sociale centrale. Au cours du XIX ${ }^{e}$ siècle, après l'abolition de la traite par l'Angleterre et avec l'implantation du " commerce légitime ", l'esclavage domestique devint un "mode de production" réservé surtout à l'exploitation agricole destinée à l'exportation (Lovejoy 2000).

Si la plupart des esclaves destinés à la traite transatlantique étaient originaires des pays frontaliers - achetés ou capturés lors d'actions de guerre -, les esclaves utilisés dans les familles ou dans les plantations étaient issus principalement de razzias menées dans des régions éloignées du Nord afin de les isoler culturellement et d'éviter des tentatives de fuite (Wendl 1999). L'origine septentrionale des esprits des esclaves d'autrefois est encore aujourd'hui évoquée lors des cultes Tchamba. Étant donné que la plupart des captifs domestiques étaient des femmes, on observe également dans l'imaginaire à l'œuvre dans la pratique rituelle contemporaine une majorité de références à des présences féminines; d'ailleurs, le vodun est souvent appelé du nom de Mami Tchamba.

Selon les affirmations que j'ai pu recueillir auprès des adeptes de Tchamba, les esclaves pouvaient être d'origine losso, kabyè, kotokoli, moba, haussa, tamberma, djougou, kante, kliko, peul et nago (yoruba ${ }^{5}$ ). Durant la transe, bien qu'on puisse faire allusion aux Nago, ce sont les expressions issues des langues du Nord les plus connues sur la côte, comme le kabyè, le peul et le haussa qui prévalent. À propos des Kabyè qui, dans le Togo actuel, occupent une région située à quatre cents kilomètres au nord de la côte, il est utile de rappeler que leur histoire a été à plusieurs reprises marquée par les contacts avec les populations du Sud, d'abord comme esclaves, puis en tant que travailleurs saisonniers (Piot 1999).

Au Togo, à la différence du Ghana et du Bénin, les responsables gouvernementaux n'ont ni élaboré des stratégies mémorielles concernant le passé de l'esclavage, ni intégré le vodun au sein d'un dispositif officiel visant la mise en valeur de la tradition ${ }^{6}$. Dans le contexte togolais, il apparaît intéressant d'analyser des discours procédant des dignitaires de Tchamba qui, par rapport aux

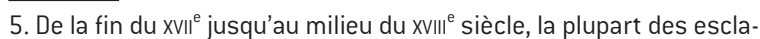
ves déportés provenaient de l'aire côtière frontalière des États d'Oyo, d'Allada et du Dahomey. Selon Paul Lovejoy (2000), pendant cette période de grande expansion de la traite, 80 à $90 \%$ des captifs étaient d'origine aja ou acculturés aja, les autres étaient d'origine yoruba. Durant la seconde moitié du XVIII ${ }^{e}$ siècle, quoique les Aja et les Yoruba constituent toujours la majorité, on enregistre un changement : les esclaves peuvent avoir des origines nupe, haussa ou de l'aire voltaïque de l'Est, venant des territoires bariba des montagnes de l'Atakora.

6. En octobre 2006, à Aného, eut lieu la première édition du Festival des divinités noires; on pouvait lire dans la brochure : « Cette manifestation vise à sauvegarder et à promouvoir le patrimoine culturel africain et de la diaspora à travers le savoir-faire pédagogique qui avait cours dans les couvents religieux traditionnels. » Cette initiative, à laquelle ont participé des personnalités déjà impliquées dans le renouveau du vodun dans la ville voisine de Ouidah, au Bénin, peut être considérée, au Togo, comme une première tentative d'ouverture aux thématiques du tourisme, du patrimoine et des échanges avec la diaspora. 


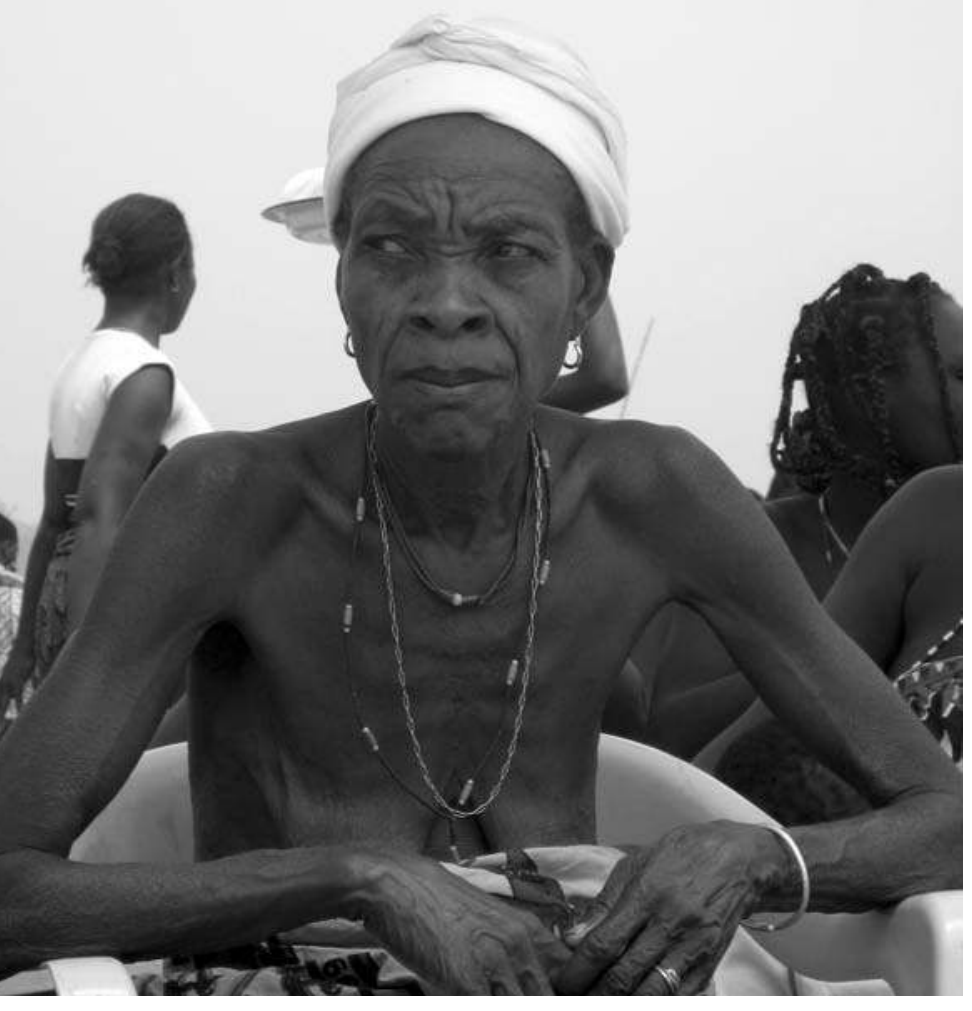

Fig. 5 Adepte de la fête nationale du vodun du 10 janvier 2006, Grand-Popo, Bénin. Photo A. Brivio.

politiques internationales contemporaines du patrimoine, occupent une position marginale. Parmi les personnalités représentatives du culte que j'ai rencontrées lors de mes enquêtes figurent en particulier Kokou Atchinou, Ablavi Laba et Edwige Agbo. Kokou Atchinou est le président du GAMAT (Groupement des adorateurs de Maman Tchamba), douanier à la retraite du port de Lomé, élu porte-parole d'une grande partie des familles qui, d'après l'expression populaire, « ont Tchamba ». Ablavi Laba, prêtresse de Tchamba impliquée dans plusieurs petites activités relevant de l'économie informelle, est âgée de soixante ans et vit à Baguida, un village situé sur la côte à quelques kilomètres de Lomé. Ablavi n'appartient pas au GAMAT, mais à un autre groupe de fidèles constitué d'une dizaine d'« autels ». Elle est aussi une Mami, c'est-à-dire une prêtresse du vodun Mami Wata qui aurait été choisie par Tchamba comme gardienne de l'autel familial. Enfin Edwige Agbo, ma troisième interlocutrice, est une riche femme d'affaires âgée de quarante ans qui vit au Bénin, à Cotonou, où elle gère un restaurant et des activités dans le grand marché de Dantokpa. Chef de culte de Mami Wata et pratiquante de Tchamba, elle ne possède pas un autel de ce vodun mais reconnaît que sa famille a été impliquée dans le commerce esclavagiste.

Au cours d'un de nos entretiens, Ablavi Laba a affirmé sans aucune réticence que sa grand-mère (togbe) était une esclave :

À une époque, nos grands-pères achetaient des esclaves qui travaillaient dans les champs et dans les maisons. Avec le temps, certaines femmes esclaves sont devenues leurs épouses et les mères de leurs enfants. Après la mort des personnes achetées, il peut arriver que les esprits, même beaucoup plus tard, demandent à avoir un autel dans la maison. D'abord on voit des signes de cette demande, par la suite on comprend qu'il faut [pour le bien de la famille] installer un autel pour eux.

Tchamba est un vodun composé d'entités censées correspondre à l'origine ethnique des esclaves décédés et des divinités que les captifs auraient pu adorer dans leur pays. Il s'agit d'un culte collectif qui concerne des familles ayant participé au commerce des esclaves ou exploité leur travail. Il est probable qu'il est né comme un culte consacré aux esclaves domestiques et plus particulièrement aux ancêtres femmes de condition captive ou servile, c'est-à-dire des concubines qui intégraient la famille de leurs maîtres. Dans les explications avancées par les pratiquants de Tchamba que j'ai rencontrés, on fait souvent allusion à une ancêtre esclave qui aujourd'hui serait revenue, sous forme d'esprit, chez ses descendants pour leur demander une place parmi les divinités familiales. Ablavi Laba affirme ainsi :

Ma grand-mère avait de l'ivoire sur ses bras bien avant qu'elle ne fût achetée. Le nom de sa famille était Banfo, elle était tamberma, originaire de Kante ; elle avait un bracelet d'ivoire parce qu'elle appartenait à un lignage royal. Avant de mourir, elle a enlevé son bracelet. Pendant des années, dans la famille, nous avons consulté des devins pour comprendre qui devait s'occuper de l'autel, mais on n'arrivait jamais à savoir. Par la suite, à travers la maladie, on a compris que c'était à moi, et maintenant c'est moi qui porte son bracelet.

De nos jours, parmi la population ewe-mina, nombreux sont ceux et celles qui revendiquent le fait d'avoir des aïeux esclaves (Rosenthal 1995). On observe simultanément une tendance à souligner, tout en assumant cette généalogie, les richesses dont leur famille esclavagiste était dotée, la beauté de la captive qui devint concubine ainsi que la fortune accumulée par celle-ci qui, à son tour, s'adonna à l'achat et à la vente d'escla- 
ves. Dans ce discours, le genre, toujours féminin, de l'ancêtre esclave en question semble faciliter la valorisation de cette partie hybride et controversée du passé, même si au cours du rite on accueille, sans distinction, les esprits de tous les hommes et de toutes les femmes qui furent victimes du commerce négrier. En ce sens, le culte dépasse le cadre des divinités familiales et change d'échelle historique et spatiale pour se décliner aujourd'hui, selon les adeptes de Tchamba, comme un rituel évocateur de la traite transatlantique. Les fêtes organisées périodiquement en l'honneur de Tchamba permettent le retour des esprits de tous les esclaves, qui se font reconnaître, parlent leur propre langue, dansent leur propre musique. En rentrant dans les corps des adeptes (trosi), le vodun donne lieu à une sorte de Babel, une cartographie imaginaire et sonore des peuples qui ont été les plus affectés par l'esclavage.

En janvier 2007, à Lomé, j'ai assisté à une cérémonie organisée par le GAMAT. Au cours de la matinée, la famille impliquée et les chefs du culte Tchamba des autres familles invitées se sont recueillis autour de l'autel. L'hôte,
Salifou Tupi, avait exposé les problèmes qui l'obsédaient, parmi lesquels ses difficultés avec l'une de ses deux femmes, très probablement victime de sorcellerie, ainsi que, dans son travail de petit entrepreneur en bâtiment, celles causées par la malveillance et la " jalousie » d'une partie de sa famille. À leur tour, tous les présents donnèrent leur avis, avançant des hypothèses et proposant des suggestions ou des remèdes. Lorsqu'un des frères cadets de Salifou s'adressa au vodun, lui demandant avec virulence pourquoi il n'accomplissait pas quelque chose de positif pour ses proches, sa rage fut tout de suite contenue par les présents qui lui rappelèrent la nécessité de rester calme face aux divinités. Les requêtes auprès de Tchamba et des autres vodun se poursuivirent lorsque tout le monde se réunit pour les prières autour de l'autel. Avant les offrandes de boisson et de nourriture cuite, trois moutons, un blanc, un noir et un rouge, furent sacrifiés aux divinités composant Tchamba. Selon un de mes interlocuteurs, on pouvait, d'après la couleur des animaux, classer les origines des esprits des esclaves convoqués à la cérémonie.

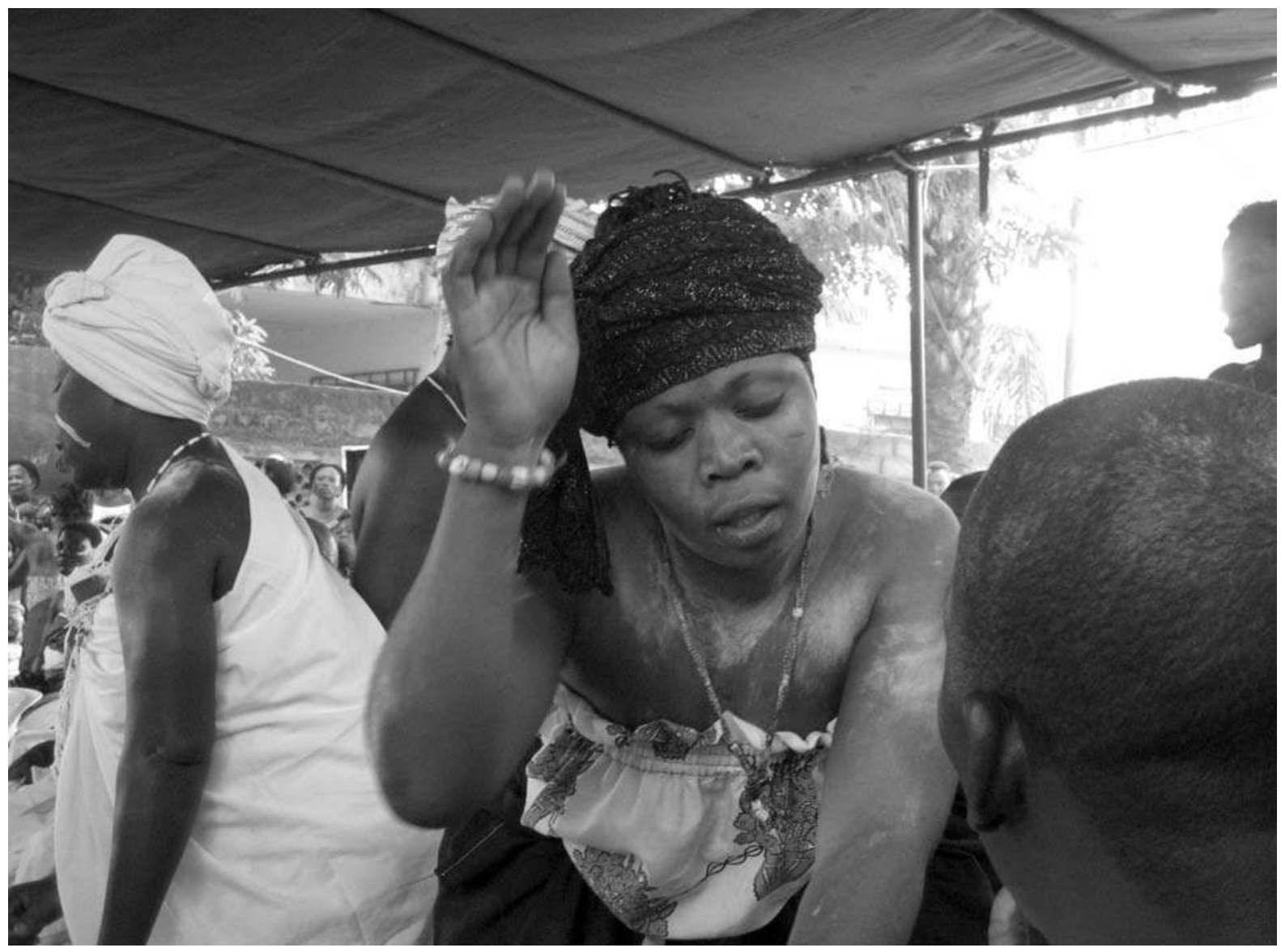

Fig. 6 Trosi de Tchamba pendant le kpeta, Lomé, Togo, 2006. Photo A. Brivio. 
La seconde partie de la cérémonie commença lentement. Les participants étaient plutôt distraits ou somnolaient - la chaleur y était pour quelque chose-, mais vers quatre heures de l'après-midi, sous la grande tente installée dans la rue adjacente à la maison, les premiers esprits d'esclaves " arrivèrent ", accompagnés par la musique. Avec une violence supérieure à celle des vodun ancestraux que j'avais rencontrés pendant les cérémonies dédiées à Dan, Sakpata ou Heviosso, ils possédaient les adeptes, dont la plupart étaient des femmes. Les corps semblaient vouloir éclater à travers des mouvements brusques et soudains, apparemment incontrôlés et convulsifs, qui, dans certains cas, allaient jusqu'à l'automutilation. L'énergie de leurs gestes semblait irrésistible, les membres se tendaient, les bustes fléchissaient et prenaient des postures antinaturelles, comme si les esprits cherchaient à se faire une place dans les corps qui les accueillaient. Kokou Atchinou me disait : "Ce sont des esprits très chauds. » On tentait de calmer leur impétuosité avec de l'eau et de les honorer avec du gin alors qu'ils dansaient en saluant le public. Dépossédés de leur identité ordinaire, les adeptes endossaient les costumes des esclaves. Dans une sorte de vestiaire, des femmes les habillaient avec beaucoup de soin et d'imagination; elles étaient chargées de choisir les vêtements, les couleurs destinées à chacun des esprits ainsi que les scarifications dessinées au kaolin sur leur visage. Kokou me présentait les « nouveaux arrivés » : « Regarde, celuilà est Bublume, il parle kabyè, souvent il est agressif... Ceux-ci sont habillés en jaune puisqu'ils sont Iedi et sont plus gentils. » La fête dura jusqu'au coucher du soleil, lorsque Kokou ordonna aux musiciens d'arrêter la musique. Les esprits, en colère à travers les adeptes qu'ils possédaient, demandèrent à prolonger la cérémonie, mais Kokou, inflexible, rappela à tous qu'il n'avait eu l'autorisation que jusqu'à six heures du soir.

\section{Le récit des origines}

Dans les années 1950, le père de Kokou Atchinou, originaire de Keta, s'est installé avec les vodun de sa famille dans le quartier de Bè, noyau historique autour duquel la ville s'est développée, pour y travailler comme maçon. À la mort de son père, et après avoir connu des problèmes financiers et de santé, Kokou s'est impliqué dans le vodun et a été initié à la pratique de Tchamba dans la maison Ouaoua, le plus important vodunnon de Lomé à l'époque, autour de laquelle une "grande famille " d'adeptes motivés par des exigences comparables aux siennes s'était formée.
En tant que président du GAMAT, Kokou a élaboré un discours semi-officiel pour expliquer et justifier l'origine de son vodun. Pourquoi l'esclavage? Pourquoi les ancêtres ont-ils été impliqués dans ce commerce que tout le monde condamne aujourd'hui? Pour répondre à ces questions, il s'est approprié certains récits mythiques qui circulent en Afrique de l'Ouest. Au cours des entretiens que j'ai eus avec lui, il m'a transmis le récit suivant, relatant l'avènement de l'esclavage en Afrique.

À l'époque des ancêtres, l'argent n'existait pas; on allait au marché pour échanger les aliments, maïs, huile rouge, igname. Mais ce système avait des problèmes, parce que ceux qui n'avaient rien à échanger ne pouvaient pas faire le marché; c'est pour cela que les ancêtres ont inventé les cauris. Où pouvait-on en trouver? Jamais sur la plage, mais dans la mer, à de très grandes profondeurs. Comment pouvait-on arriver jusqu'au fond de la mer? Les chasseurs qui traversaient la forêt et faisaient peur à tout le monde ont commencé à chasser des hommes. Avec l'aide des pêcheurs qui habitaient sur la côte, ils emmenaient les hommes capturés au large, sur des bateaux, ligotaient leurs mains et leurs pieds, leur attachaient des pierres au cou et les faisaient descendre dans l'eau. Après quelques heures, ils récupéraient leurs corps remplis de cauris; ils enlevaient les cauris et, après s'être déplacés un peu, ils lançaient à nouveaux les noyés dans la mer où les corps se décomposaient. C'est comme ça que l'esclavage a commencé. Les pêcheurs revenaient sur la côte, ils avaient de l'argent et prenaient d'autres esclaves. Après, il y en a eu d'autres qui sont arrivés d'Amérique pour acheter les personnes, mais l'origine est celle-ci.

Cette narration évoque une époque immémoriale caractérisée par le troc et l'absence de l'argent, qui aurait pris fin avec l'invention des cauris. Il s'agirait d'une période d'innocence et de pureté, qui n'aurait pas encore été "contaminée " par l'économie de marché. Mais le troc bientôt faillit et les hommes entrèrent dans le circuit vicieux de l'argent. Kokou m'expliquait qu'au temps des ancêtres il n'y avait rien à acheter, pas de voitures, pas de maisons, pas de terres. Acheter des esclaves était la seule solution pour investir l'argent et accumuler des richesses.

Pour ramasser les cauris au fond des eaux marines, des esclaves auraient donc été utilisés par les ancêtres comme des filets : ils étaient ligotés, attachés à des pierres et jetés dans l'océan; leurs corps, pleins de cauris, étaient ensuite "pêchés ". Ce genre de narration chargée d'une extrême violence semble illustrer un degré zéro de la morale économique : l'équation entre les cauris et les corps humains signifierait que l'enrichissement 
ne peut se faire qu'au détriment de la vie d'autrui'. Dans le mythe, la monnaie (les cauris) vampirise la chair des esclaves. Leur mort est une métaphore de l'hommeobjet, devenu outil de travail. La mort en mer semble être aussi une métaphore de la traite négrière, c'est-àdire de la disparition des esclaves, « noyés » dans l'océan, prisonniers des navires-cauris qui les ont engloutis : la mer était un lieu de mort à la fois réel et imaginaire. À travers la représentation de l'homme-appât, c'est la déprédation opérée par le commerce transatlantique qui retentit brutalement sur l'imaginaire tragique des origines de la modernité en Afrique. Ces origines sont identifiées à un marché déjà globalisé, avec ses flux et reflux de richesses, de domination et d'exploitation, où le capitalisme agissait avec toute sa cruauté (Shaw 2002). Les mêmes narrations dérangeantes emplissent les représentations contemporaines de l'Afrique «blessée " par l'économie de marché et par les mirages de la globalisation.

Pourtant, cette appréhension collective de l'économie matérielle et symbolique de l'esclavage, transmise à travers les mythes, ne suffit pas à saisir l'ambivalence des perceptions morales qui affectent un imaginaire impliqué dans le commerce négrier. Cet imaginaire est encore pris dans les filets des représentations historiques de l'argent :

Tchamba a commencé à se manifester après l'abolition de l'esclavage, le 27 avril $1848^{8}$ [...]. Après la mort de grand-père, les difficultés ont commencé pour toute notre famille; la vie est devenue trop difficile; alors on s'est demandé : pourquoi cette malchance? Comme ça, nous avons découvert qu'il fallait faire des cérémonies pour les personnes qui avaient été achetées pendant le commerce des hommes.

En fait, après l'abolition de l'esclavage, l'endettement serait devenu progressivement un problème majeur dans les familles anlo de la côte, auxquelles l'ancêtre de Kokou appartenait (Greene 1996). C'était la fin d'une époque faste pour certaines familles qui avaient profité de la traite pour s'enrichir. L'argent évoque le prestige et le pouvoir des aieux dont, à travers le vodun Tchamba, les descendants actuels essaient de s'emparer à nouveau. Loin des images vampirisées, les cauris évoquent aujourd'hui la puissance mystique. On les trouve dans la plupart des rituels et des objets vodun, symboles d'une richesse et d'un pouvoir immatériels. La même ambivalence est valable pour la mer, à la fois dévoratrice d'hommes et royaume des divinités de la richesse et de la modernité, vodun les plus adorés dans la région ${ }^{9}$.
La pratique du culte reflète la même ambiguïté car si, d'une part, elle avance une critique liturgique implicite de l'économie «immorale » de l'esclavage en tant que phénomène historique d'une époque à jamais révolue, de l'autre, elle vise à transformer sa mémoire en ressource spirituelle et financière. Dans cette pratique de recontextualisation du passé négrier, la valeur monétaire des cauris revêt une efficacité symbolique censée produire l'abondance et la fécondité.

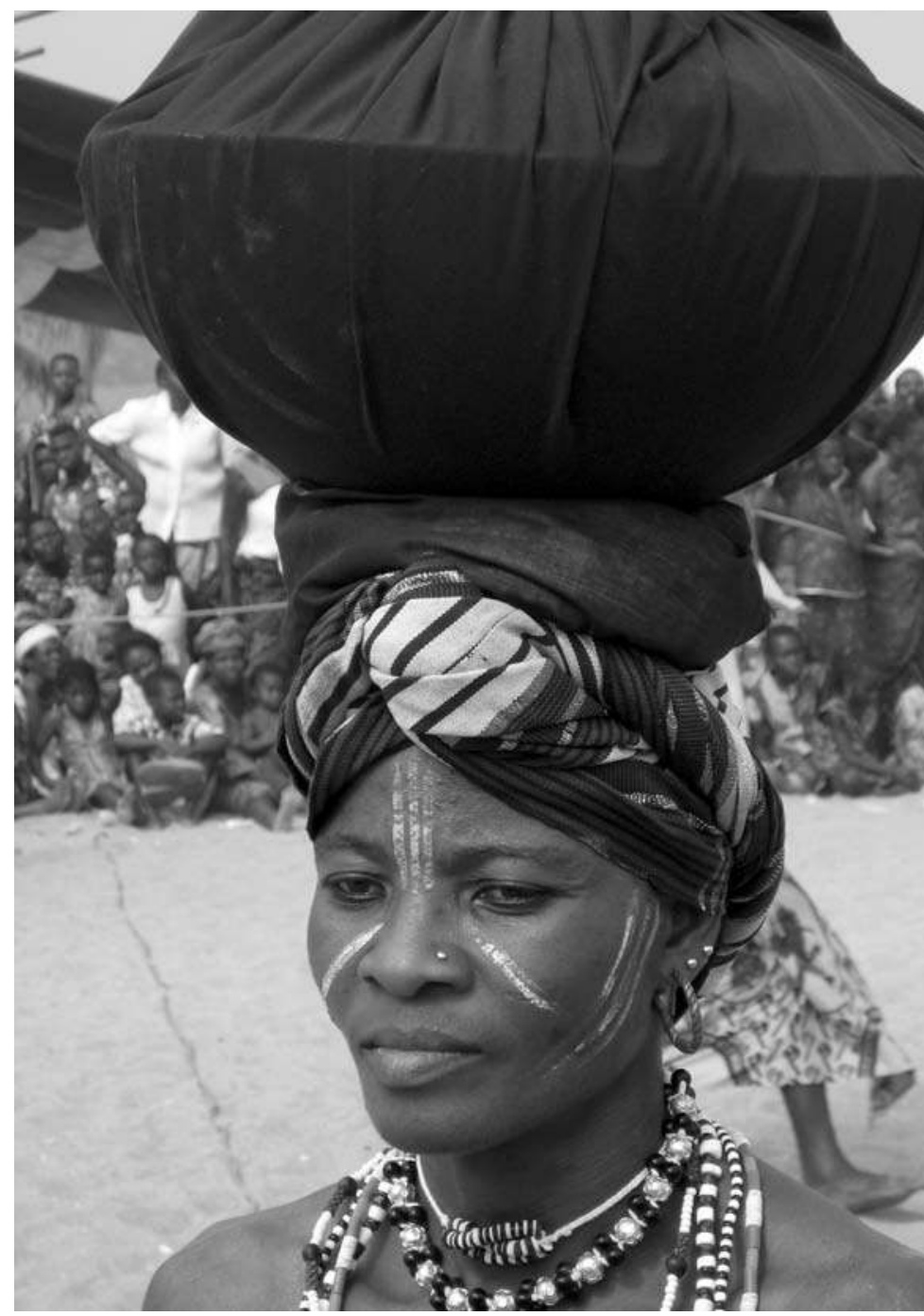

Fig. 7 Trosi de Tchamba lors de la fête nationale du vodun du 10 janvier 2006, Grand-Popo, Bénin. Photo A. Brivio.

7. Des versions comparables de cette légende ont été recueillies et analysées par d'autres chercheurs, voir Austen 1993; Ferguson 1999; pour des sociétés non africaines : Taussig 1980; Wachtel 1992.

8. Le 27 avril 1848, la République française publia le décret d'abolition de l'esclavage dans les colonies françaises.

9. Je me réfère aux vodun liés à Mami Wata. 


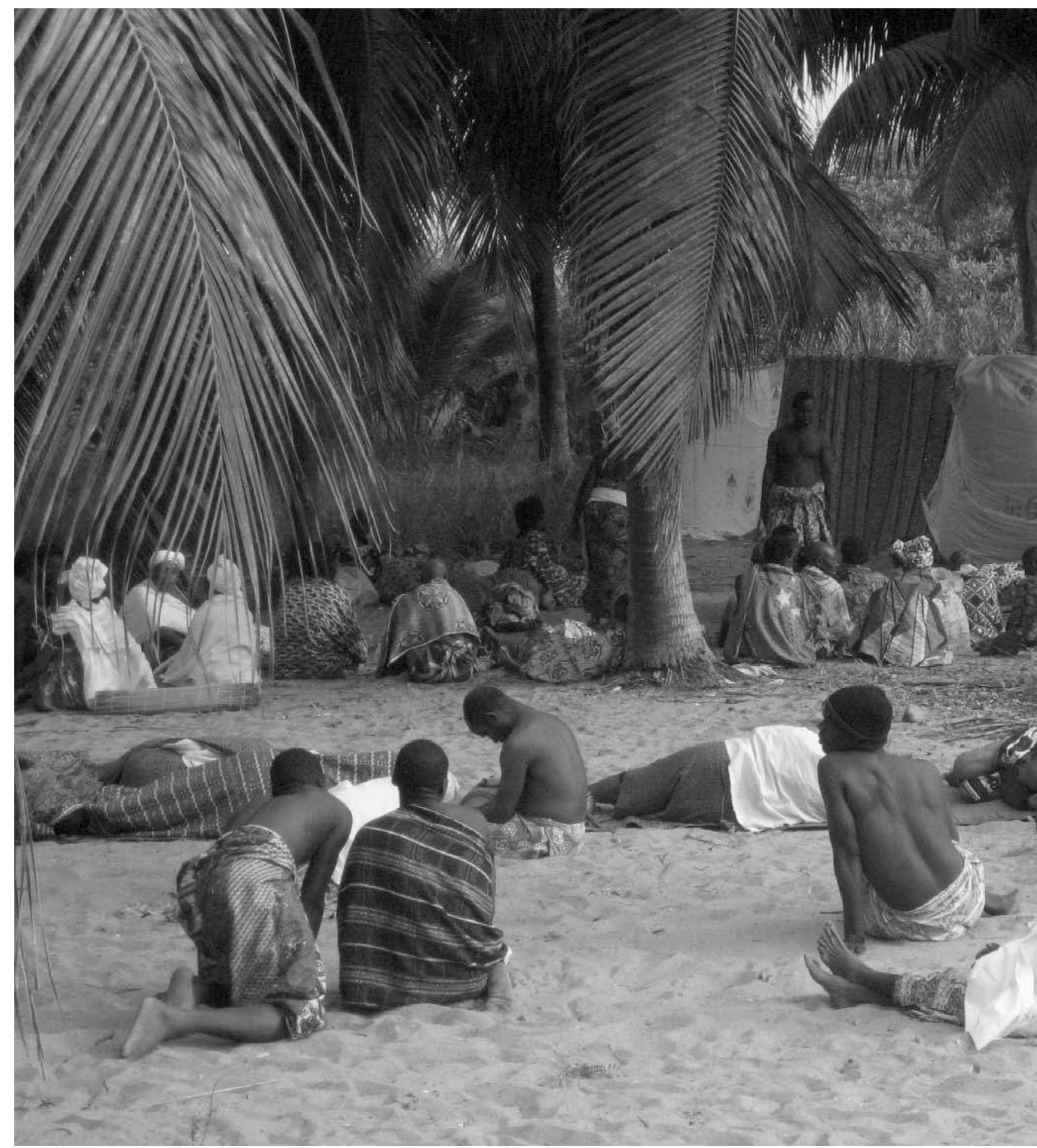

Fig. 8 À l'extérieur du dzogbe, Grand-Popo, Bénin, 2006. Photo A. Brivio.

D’après Kokou Atchinou, Tchamba est « le signe de ce que nos ancêtres ont fait ». Ce signe est donc simultanément négatif et positif, signe de mort et de violence, de richesse et de prospérité. Ainsi, à travers Tchamba, les descendants de ceux qui ont été impliqués dans le commerce négrier incorporent une pensée rituelle de leur histoire personnelle et sociale. L'apparition de Tchamba au sein des familles imprime dans les mémoires un passé qui n'est nullement renié ou mitigé, mais assumé avec fierté. Ce vodun est considéré comme pou- 


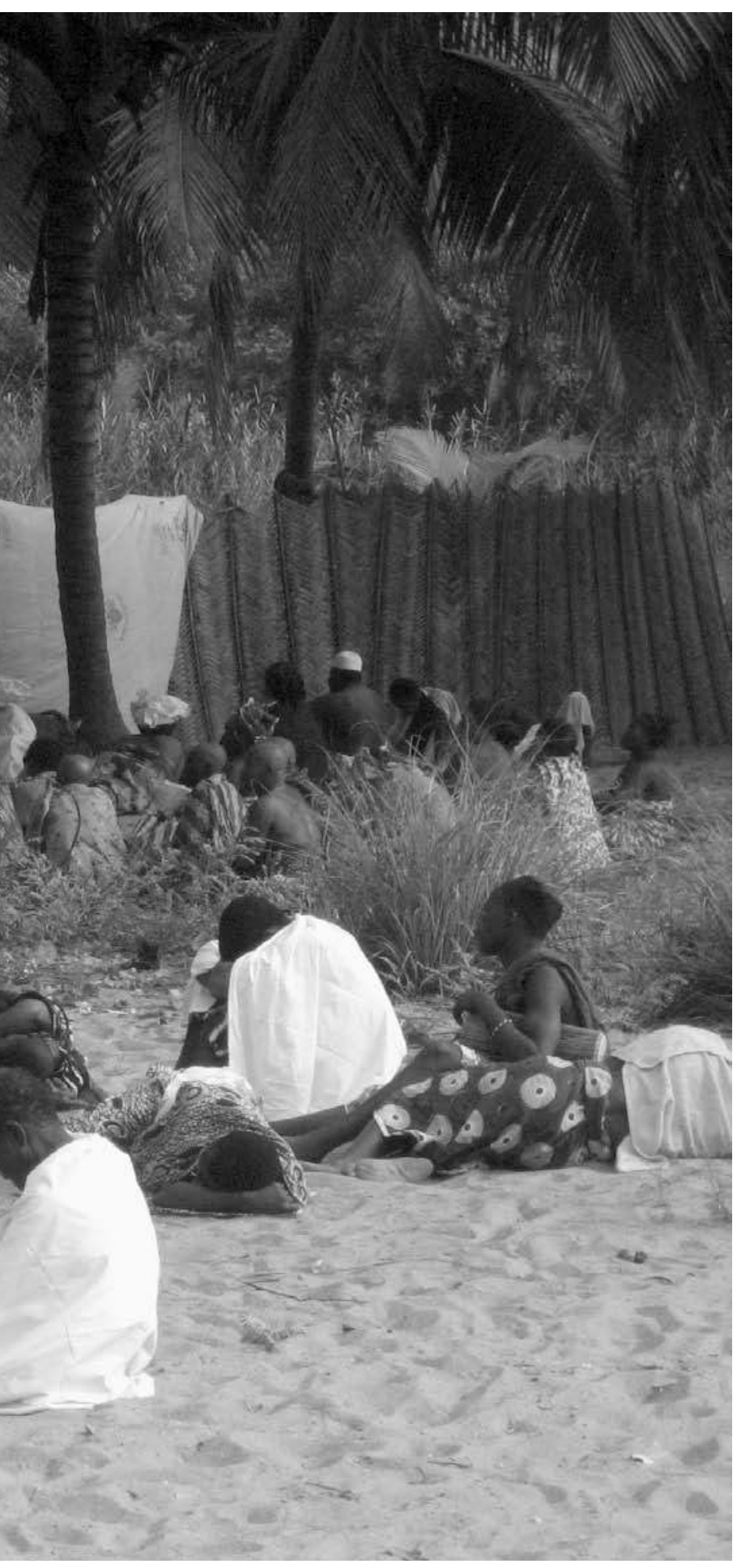

vant être particulièrement puissant et dangereux : «être de son côté " est la marque d'une position sociale et spirituelle privilégiée. Tout comme dans le passé, l'esclave devenu esprit est un porteur potentiel de richesse et de bien-être. Périodiquement, les membres de la famille
" qui ont " Tchamba se réunissent autour de l'autel, s'agenouillent et font leurs requêtes. L'esclave demeure un bien précieux, une ressource pouvant également inspirer un sentiment de peur, la crainte d'une menace invisible.

\section{Lieux et objets de l'imaginaire du Nord}

Les traces de mort et de violence que le récit de Kokou Atchinou rend explicites sont également présentes dans la pratique et l'iconologie du culte. L'autel de Tchamba est constitué de plusieurs récipients censés représenter les diverses origines ethniques des esclaves. Près de ces ustensiles, il y a le tabouret-siège de l'ancêtre (togbesibi) qui a été impliqué dans le commerce ou de la "Mami ", la femme esclave ancestralisée et donc intégrée dans la famille. L'autel est également formé de cordes, de chaînes, de fers et d'un certain nombre de cauris et de petites perles en verre. L'ensemble des objets, visibles seulement lors des cérémonies, est disposé d'une manière telle que, recouverts d'une toile, ils évoquent la figure du défunt. Le corps appartient à celle ou à celui qui n'a pas été enterré selon les usages de son peuple parce qu'il est mort loin de sa terre, de ses ancêtres, de ses divinités.

Comme le rappelle Wendl, « dead slaves were buried without any particular funeral rites outside the villages, in the wilderness [...]. In Mina funeral rites, the dichotomy of "village" and "wilderness" is used as a metaphor for the social centre, on the one hand, and for the social periphery on the other hand " (1999: 114). Selon Albert de Surgy, en milieu ewe ceux qui n'ont pas eu droit à des cérémonies funéraires ne peuvent accéder au rang d'ancêtre : «Si ces cérémonies tardent à avoir lieu, ils ne cessent de tourmenter les personnes qu'ils en jugent responsables, se manifestant parfois à elles sous forme de fantômes, jusqu'à les décider à leur donner une sépulture convenable » $(1988: 125)$. En reprenant ces remarques, nous pouvons conclure que, dans le culte Tchamba, le corps de l'esclave abandonné à une mort sans cérémonie impose un dialogue à la famille au sein de laquelle il a vécu. Ainsi, il sort de l'oubli auquel sa condition de captif l'avait condamné. Les esclaves décédés, privés de rituels funéraires, appartiendraient donc à la même catégorie que celle des «mauvais morts ", c'est-à-dire ceux qui ont quitté la vie d'une manière soudaine et prématurée, généralement suite à une agression sorcellaire ou à la vengeance d'un vodun. À travers la maladie, la malchance, la mort, ces esprits non pacifiés reviennent déranger leur famille. Cependant, par 
la divination, les vivants peuvent apprendre la cause de ces désordres et y remédier en organisant des cérémonies ou, comme dans le cas de Tchamba, en fabriquant un autel. Les corps des mauvais morts étaient enterrés dans le dzogbe, terme indiquant un «lieu du feu ", en brousse ou en tout cas aux marges d'un village ${ }^{10}$. Selon plusieurs récits recueillis auprès des populations ewemina, les esclaves enterrés dans le dzogbe sont comme les mauvais morts condamnés à errer dans la nature, privés de la possibilité de devenir ancêtres et de réintégrer le cycle de la vie.

Lors de l'installation du vodun Tchamba, un rituel considéré comme fondamental a lieu dans le dzogbe, dans un espace clôturé où seuls sont admis les chefs de culte et les initiés; les autres demeurent pendant des heures, parfois la nuit durant, à l'extérieur de l'enceinte. Les adeptes de Tchamba, en recomposant un corps symbolique, sont censés accueillir les esprits abandonnés de tous les esclaves. Certains parmi eux s'imposent comme des individus identifiables, d'autres, la majorité, sont plutôt assimilés à une appartenance ethnique présumée. Presque toujours, à côté de Tchamba un autre vodun, nommé Adela, agit. Il accueille les esprits des chasseurs et des guerriers morts en brousse ou en bataille. À cet égard, de Surgy remarque : "Tous ceux qui ont péri par le fer ou en relation avec la puissance du fer (Gu) qui les animait ou les emportait, que ce soit à la chasse, à la guerre ou dans un accident d'automobile [...] se transforment en Adela, c'est-à-dire en "chasseurs", nom qui leur est donné du fait de leur errance isolée dans l'univers de brousse... » (1988:131).

J'ai recueilli deux versions expliquant la présence d'Adela à côté de Tchamba. Selon Kokou Atchinou, celleci est due à la nécessité de célébrer un ancêtre chasseur : «Mon grand-père était chasseur, il partait à la chasse et s'il ne trouvait pas d'animaux, il prenait des hommes. Et les gens ne fuyaient pas, parce qu'on n'échappe pas à quelqu'un qui est armé. C'est pour cela que les grands commerçants étaient souvent aussi des chasseurs. " Mais selon Laba Ablavi, plus proche de de Surgy : «Ceux qui sont morts d'une manière non naturelle vont chez Ade et Adela. Adela est le vodun de ceux qui sont morts à la chasse ; Ade est celui de tous ceux qui sont morts d'une manière non naturelle, par exemple les esclaves qui sont morts sur le chemin qui les emmenait à la côte. "

Si Kokou Atchinou semble adhérer à la représentation de la mémoire héroïque des maîtres ancêtres, Laba Ablavi, qui affirmait au contraire sans problème que sa " grand-mère " était une esclave, est plus sensible au destin des victimes : nous retrouvons ici l'ambiguïté du culte Tchamba où les esclaves sont à la fois des ancêtres familiaux et des victimes. D'ailleurs, les récits cachent, dans l'imaginaire de la chasse, un second niveau d'ambiguité. En se souvenant, à travers le rituel, à la fois des gestes de l'ancêtre chasseur qui affronte les dangers de la brousse et du sort de ses victimes, parmi lesquelles se trouvaient des bêtes, des humains et des esprits pouvant être " tués " par erreur lorsqu'ils se dissimulaient sous des formes animales ${ }^{11}$, la présence $d u$ vodun Adela semble confirmer le regard méprisant des patrons envers les esclaves. Si l'espace sauvage est traversé par des entités qui échappent à la classification, cette polyvalence se répercute aussi sur le statut des hommes chassés pour être vendus comme esclaves. Bien qu'appartenant désormais à une condition inférieure, ces hommes pouvaient avoir une emprise spirituelle avec laquelle, encore aujourd'hui, les adeptes de Tchamba négocient et qu'ils tentent de contrôler. Dans un article récent, John Parker analyse ce qu'il définit comme le « Northern gothic », c'est-à-dire l'aura exotisante que les peuples akan ont construite autour des populations de la savane septentrionale. Cette image "gothique " attribuée à des sociétés perçues comme "primitives " baignant dans un monde traversé par des forces surnaturelles aurait accompagné l'action et le regard guerrier des razzieurs du Sud. En ce sens, d'après Parker, il y a eu une perspective hégémonique, fondée sur une conception centralisatrice de l'organisation politique et militaire, qui aurait nourri l'imaginaire à l'œuvre dans la rencontre culturelle africaine entre conquérants et captifs. Au cours de nos entretiens, Kokou décrivait la chasse comme le seul système d'approvisionnement d'esclaves; les chasseurs se rendaient dans les villes et livraient leurs « proies » aux grands marchands, tels les ancêtres d'Ouaoua, qui " attendait dans sa maison que les chasseurs lui apportent les esclaves ». La superposition entre animaux et captifs que l'idée de proie véhicule explicite une représentation de l'homme du Nord aujourd'hui encore dénoncée au sein de la population kabyè au Togo (Piot 1999; Koffi 2000). Mais le contrepoids symbolique de cette perception suggère que ces mêmes peuples infériorisés au cours de l'histoire seraient les grands connaisseurs d'un monde et d'un paysage traversés par des phénomènes occultes. La place pré-

10. Selon Palau-Marti (1993), au Bénin, « dans certains cas, et notamment pour les "morts tragiques", on fait l'enterrement en brousse et sans jamais signaliser la tombe, tout le contraire. On porte ainsi loin du village les victimes de la foudre, les gens "qui toussent", les femmes mortes en couche $\gg$.

11. Cette possibilité a été évoquée par Edwige Agbo lors d'un entretien. 
pondérante de l'invisible dans la culture vodun communique rituellement avec la fascination pour les régions du Nord censées être les hauts lieux d'un ésotérisme barbare. Claude Meillassoux remarquait que le racisme du maître se construit aussi sur l'exotisme de l'esclave en tant qu' « étranger absolu » (1986: 68). En effet, durant les cérémonies célébrant Tchamba, le "Nord » est mis en scène à travers des images, des objets et des gestes provenant d'un imaginaire où les noix de cola, les instruments musicaux, les bijoux, les théières, les peaux d'animaux et les sacs en cuir finissent par constituer les pièces à conviction d'une sorte d'" ethnographie du Nord » (Wendl 1999 : 116). Les voiles colorés, les casaques endossées sur des pantalons longs, les turbans, les fez et les chapelets pour la prière portés comme s'ils étaient des colliers ou des bandeaux ceignant les têtes, les bracelets en fer ou en ivoire sont des éléments du déguisement à travers lequel les adeptes en transe évoquent le monde de la savane septentrionale. Les peintures que l'on peut souvent voir sur les autels de Tchamba montrent une femme peule, idéal exotique de beauté et d'élégance, dont les postures et l'accoutrement sont mimés lors du rituel.

Au cours de la liturgie, cette inversion des rôles a pour but de satisfaire les requêtes des esprits ; ainsi, elle semble œuvrer à la création d'un espace dialogique entre les époques et leurs acteurs. Cette fête permet aux esprits des esclaves de sortir de leur solitude. Leur ombre menaçante, faite de mort, de souffrance et de peur, se dissipe à travers le rituel de la réunion collective. Néanmoins, dans le culte Tchamba, le mépris du maître envers l'esclave demeure dans toute sa polysémie. La cérémonie représente des valeurs esthétiques évidentes dans le soin avec lequel les adeptes, dont la majorité est constituée de femmes, sont habillés. On assiste à une très forte participation émotionnelle, à des formes de ravissement collectif qui souvent transcendent les niveaux habituels qu'on peut observer dans d'autres rituels vodun. On pourrait parler d'un véritable désir ou d'une passion pour l'Autre, jusqu'à dire que «there is love and splendid admiration now for the amefefle, idealized in retrospect, if ever there was contempt" (Rosenthal 1998 : 112). Le dévouement montré par les adeptes à travers leurs expressions corporelles entre en résonance avec le devoir de prodigalité des organisateurs de la fête. À travers le nombre d'animaux sacrifiés et les boissons offertes aux nombreux invités, les familles «qui ont " Tchamba accueillent les esprits du Nord, beaux et impétueux. Pourtant, la générosité et l'admiration dont ces esprits sont les destinataires ne semblent pas exclure une sorte de détachement, rappelant par là le regard qui se veut encore dominant du maître. Cela dit, le désir et l'ardeur demeurent les moteurs de la rencontre entre l'homme et le vodun en s'inscrivant dans une logique caractérisée par une tolérance " raciale » envers les divinités des autres, davantage liée à la nécessité et à la peur qu'à la compassion.

\section{Conclusion}

À plusieurs reprises, mes interlocuteurs m'ont communiqué leur conviction que l'abolition de l'esclavage n'avait pas mis fin à celui-ci. En disant cela, ils faisaient allusion à des situations contemporaines dans le monde, mais aussi aux cas récents, divulgués entre autres par la presse internationale, d'enfants esclaves au Togo et au Bénin. Edwige Agbo, en expliquant les modalités à travers lesquelles Tchamba peut se manifester, m'a raconté son conflit avec l'une de ses jeunes employées domestiques du Nord (vidomègon, littéralement « enfant qui est chez autrui » en langue fon) :

Elle ne travaillait pas, parfois elle restait allongée pendant toute la journée. Moi je gardais le calme. Un jour, elle me disait "je vais partir » ; un autre, "je resterai ici jusqu'à ma mort ». Moi, je n'étais pas sûre qu'elle soit un esprit ou un être humain. Un matin, en se levant, elle m'a dit : « Donne-moi l'argent, je vais partir »; elle est partie et m'a laissé la bague de Tchamba. Elle était Tchamba elle-même.

L'incertitude sur l'identité de la vidomègon qui, d'après Edwige Agbo, était peut-être une jeune femme, ou peutêtre un esprit, avait empêché la « maîtresse » de s'opposer à la requête de sa domestique. En relatant cette histoire, mon interlocutrice reconnaissait implicitement que les "enfants placés " sont des esclaves modernes incarnant l'ambiguïté identitaire qui fut celle des anciens esclaves du Nord. Selon son interprétation, la vidomègon serait donc arrivée du Nord afin de l'intimider et de lui rappeler ses devoirs envers Tchamba. Tout en n'exprimant aucun jugement moral et sans faire aucune allusion explicite aux formes d'esclavage contemporain, Edwige Agbo percevait une menace réelle provenant de ces esprits " qui peuvent arriver et te faire du mal, puisque personne ne peut vraiment savoir ce qu'ont fait tes parents dans le passé" .

Dans le langage des adeptes du vodun Tchamba, le drame de l'esclavage s'exprime par des images de déracinement, de rupture d'une continuité ancestrale et in fine met en scène la solitude de l'homme arraché à sa famille. Si les adeptes sont conscients de la tragédie 
physique et spirituelle qui fut imposée aux captifs d'antan, cette conscience apparaît désormais comme un reflet de leurs peurs actuelles. Les familles des anciens commerçants d'esclaves qui " ont Tchamba " sont disposées à calmer les inquiétudes des esprits en les intégrant dans leur communauté. Cette assimilation est ambivalente : si elle évoque inévitablement la sujétion et l'altérité de l'esclave, elle le hisse simultanément au niveau d'un vodun, c'est-à-dire qu'elle lui confère un pouvoir auquel il faut se soumettre. Une très grande indétermination est présente également dans l'identité des adeptes de Tchamba eux-mêmes, descendants en ligne paternelle de riches marchands d'autrefois, mais ayant souvent aussi du sang esclave. Cette altérité, qui est surtout féminine, assimilée au sein du lignage et domestiqué par l'histoire, retrouve sa singularité esthétique lors du rituel. Grâce au culte Tchamba et à ses logiques multiples, les familles dont les aïeux ont été impliqués dans la traite se fraient un chemin pour arbitrer les conflits entre les différentes composantes du lignage et, au-delà de la sphère familiale, pour aborder des mémoires socialement difficiles à assumer.
Il n'y a ni sentiment de culpabilité ni recherche d'une rhétorique explicite dans les discours des fidèles, où se manifeste plutôt l'exploration de la complexité et des contradictions du monde visible et invisible, de la vie passée et contemporaine. Cette " conscience historique " retentit encore sur le présent, là où l'oubli n'est pas pensable puisque le passé a été incorporé; là où ce dernier a pénétré dans les profondeurs des consciences individuelles à travers un langage fait de peurs, de désirs et de passion.

mots clés / keywords : esclavage // slavery • Bénin // Benin - vodun // vodun . Tchamba // Tchamba • mémoire // memory $\cdot$ possession // possession.

Département de sciences humaines «Riccardo Massa » Université Milano-Bicocca alezup@yahoo.it

\section{Bibliographie}

\section{Argenti, Nicolas}

2006 « Remembering the future: slavery, youth and masking in the Cameroon Grassfields », Social Anthropology 14(1) : 46-69.

Augé, Marc

1988 Le Dieu-Objet. Paris, Flammarion.

Austen, Ralph

1993 « The moral economy of witchcraft: an essay in comparative history », in John et Jean Comaroff (éd.), Modernity and its Malcontents. Chicago, University of Chicago Press : 89-110.

2001 « The slave trade as history and memory: Confrontations of slaving voyage documents and communal traditions », William and Mary Quarterly 58 : 229-244.

\section{BAKO-ARIFARI, Nassirou}

2000 « La mémoire de la traite négrière dans le débat politique au Bénin dans les années 1990 », Journal des africanistes $70[1-2$ ].

BodDy, Jane

1989 Wombs and alien spirits. Women, men and the zar cult in Northern Sudan. Madison, The University of Wisconsin Press.

COMAROFF, John et Jean

1992 Ethnography and the Historical Imagination. Boulder, Westview Press.

de Medeiros, François (éd.)

1984 Peuples du golfe du Bénin. Paris, Karthala.

Ferguson, James

1999 Expectations of Modernity: Myths and
Meanings of Urban Life on the Zambian Copperbelt. Berkeley, University of California Press.

GREENE, Sandra E.

1996 Gender, ethnicity and social change on the upper slave coast. A history of anlo-ewe. Londres, James Currey.

$2003 \ll$ Whispers and Silences: Explorations in African Oral History », Africa Today 50 : 40-53.

Herskovits, Melville et Frances

1933 An outline of Dahomean religious belief. Wisconsin, American Anthropological Association.

Koffi, Kodjo

2000 « Note sur le thème de l'esclavage dans la politique togolaise actuelle », Journal des africanistes 70 : 233-237. 
LAW, Robin

1991 The Slave Coast of West Africa 1550-1750. Oxford, Clarendon Press.

\section{LE HÉRISSÉ, Auguste}

1911 L’Ancien Royaume du Dahomey. Mœurs, religion, histoire. Paris, Émile Larose.

\section{Lovejoy, Paul}

2000 Transformations in slavery. Cambridge, Cambridge University press.

\section{LoVell, Nadia}

2002 Cord of blood. Londres, Pluto Press.

\section{MAupoil, Bernard}

1943 La Géomancie à l'ancienne côte des Esclaves. Paris, Institut d'ethnologie.

\section{Meillassoux, Claude}

1986 Anthropologie de l'esclavage. Paris, PUF.

\section{Mignon, Alain}

1985 La Terre et le Pouvoir chez les Guin du Sud-Est Togo. Paris, Publications de la Sorbonne.

\section{PALAU-MARTi, Montserrat}

1993 Société et religion au Bénin (les SabèOpara]. Paris, Maisonneuve et Larose.

\section{PARKER, John}

2006 « Northern gothic: witches, ghosts and werewolves in the savana hinterland of the Gold Coast, 1900s-1950s », Africa $76:$ 352-380.

\section{PieTz, William}

1985 «The problem of the fetish, | 1 , Res $9: 5-1$.

1987 «The problem of the fetish, II », Res 3 : 23-45.

\section{Piot, Charles}

1999 Remotely Global: Village Modernity in West Africa. Chicago, The University of Chicago Press.

\section{PRESTON BLIER, Suzanne}

1995 African Vodun: Art, Psychology and Power. Chicago, The University of Chicago Press.

\section{RosentHal, Judy}

1995 «The signifyng crab », Cultural Anthropology 10 [4] : 581-586.
1998 Possession, Ecstasy and Law in Ewe Voodoo. Charlottesville, The University Press of Virginia.

\section{SHAW, Rosalind}

2002 Memories of the slave trade. Chicago, University of Chicago Press.

\section{SuRGY, Albert de}

1998 Le Système religieux des Évhé. Paris, L'Harmattan.

\section{TAUssig, Michel}

1980 The Devil and Commodity Fetishism in South America. Chapel Hill, University of North Carolina Press.

\section{WAchtel, Nathan}

1992 Dieux et vampires : retour à Chipaya. Paris, Seuil.

\section{WendL, Thomas}

1999 « The Tchamba cult among the Mina in Togo », in Heike Behrend et Ute Luig (éd.), Spirit possession: modernity and power in Africa. Madison, The University of Wisconsin Press : 111-123.

\section{Résumé / Abstract}

Alessandra Brivio, « Nos grands-pères achetaient des esclaves... » Le culte de Mami Tchamba au Togo et au Bénin. L'article se propose d'aborder, à travers l'analyse du vodun Tchamba, la mémoire de l'esclavage partagée par les adeptes du culte, chez les populations de langue ewe et mina des aires côtières du Ghana sud-oriental, du Togo et du Bénin.

Tchamba est le lieu ou les esprits des esclaves et ceux des maîtres se rencontrent. Sur l'autel, on célèbre soit les ancêtres impliqués dans le commerce d'esclaves, soit leurs victimes, les esclaves intégrées dans la famille. Les cérémonies collectives, au-delà de la sphère familiale, consacrent les esprits de tous les esclaves et de tous ceux qui sont morts loin de leurs terres. En suivant l'ambiguïté éthique de la pratique du vodun, cet article présente une enquête sur les imaginaires opaques à l'intérieur desquels agissent les fidèles de Tchamba; sur des lieux du sacré remémorant un passé de mort et de privation, mais aussi de passion et de désir de richesse.
Alessandra Brivio, "Our grandfathers used to buy slaves...". The cult of Mami Tchamba in Togo and Benin. - Through the analysis of the Tchamba vodun, this article considers the memory of slavery shared by cult members, among the Ewe-and Mina-speaking populations of the coastal regions of southeast Ghana, Togo and Benin.

Tchamba is the place in which the spirits of the slaves and those of the masters meet. Either the ancestors involved in the slave trade, or their victims, slaves integrated into the family, are celebrated on the altar. The collective ceremonies, beyond the family circle, consacrate the spirits of all the slaves and of all those who died far from their homeland. By following the ethical ambiguity of the practice of vodun, this article presents an investigation into the opaque imaginaries within which the Tchamba faithful act; on places of the sacred commemorating a past of death and privation, but also of passion and the desire for riches. 